\title{
A Relação Teoria e Prática na Psicologia da Educação: implicações na formação do educador
}

\author{
Luciane Maria Schlindwein
}

\begin{abstract}
Resumo
Este trabalho tem por objetivo discutir a relação teoria e prática no campo da Psicologia da Educação e suas implicações para a formação do educador, a partir da análise dos trabalhos apresentados nas reuniões anuais da ANPEd (especialmente no Grupo de Trabalho Psicologia da Educação (GT20). O estudo envolveu a leitura de trabalhos encomendados, comunicações e pôsteres, apresentados nas reuniões anuais da ANPEd, no período compreendido entre os anos de 1998 e 2009. Os estudos apresentados nesta última década são indicativos de que temos uma aplicação da psicologia na escola, que basicamente é alimentada pela Psicologia da Aprendizagem. Os estudos sobre subjetividade, identidade e constituição do sujeito indicam uma preocupação com o ser humano (constituição da subjetividade), mas ainda com o enfoque muito psicológico. Ao que parece, ainda não superamos a dificuldade de se pensar esta subjetividade em termos de contextos mais amplos. E, o que prevalece, nestes estudos, são abordagens teóricas da psicologia do desenvolvimento. Outro bloco de estudos, apoiados principalmente na incorporação de contribuições da psicologia social são indicadores de uma possibilidade de olhar/se pensar a escola. Entretanto, aqui, a limitação ainda é de ordem mais conceitual. Ou seja, a dicotomia permanece. Finalmente, identificamos uma linha que deveria ou poderia se constituir na crítica epistemológica de toda esta construção; mas que ainda está muito presa a abordagens; poder-se-ia partir de uma visão mais contextualizada, superando o debate focado em abordagens. Consideramos que é preciso pensar a psicologia para além do território de autores ou de áreas (como a psicologia da aprendizagem, do desenvolvimento). Talvez nosso movimento precise ser redirecionado, trazendo as contribuições da psicologia para uma visão mais ampla, inclusive de escola (que permita compreender a escola e seus atores em todas as suas complexas dimensões). Escola não é só espaço de aprendizagem, ou de desenvolvimento, ou de formação de professores.
\end{abstract}

Palavras-chave: Psicologia educacional, aprendizagem, formação de professores.

\section{The Relation Between Theory and Practice in Educational Psychology: implications for teacher training}

\begin{abstract}
This work aims to discuss the relation between theory and practice in the field of Educational Psychology and its implications for the teacher's formation, by the analysis of the papers presented in the ANPEd annual meetings (especially in the working group Educational Psychology - GT20). The study involved the reading of 'ordered works', communications and posters presented at the annual meetings of ANPEd, in the period between 1998 and 2010. The studies presented in this last decade are indicative of that we have an application of psychology in school that basically is fed by the Learning Psychology. Studies on subjectivity, identity and subject constitution indicate a concern with the human being (Constitution of subjectivity), but still with a too much psychological approach. It seems that we have not yet overcome the difficulty of thinking this subjectivity in terms of wider contexts. And, what prevail in these studies are theoretical approaches of developmental psychology. Another block of studies supported mainly in incorporating contributions from social psychology are indicators of a possibility to look/think about school. However, the limitation here is still of more conceptual order. I.e. the dichotomy remains. Finally, we identified a line that should or could be an epistemological critique of all these constructions; but that is still very much stuck to approaches; it could have started from a more contextualized vision, surpassing the debate that is focused on approaches. We believe that we have to think about the psychology beyond the territory of authors or areas (like the psychology of learning, of development). Maybe our movement would need to be redirected, bringing Psychology's contributions to a broader view, inclusive about school (able to comprehend the school and his actors in all its complex dimensions). School is not only a space of learning, or of development, or of teacher training.
\end{abstract}

Keywords: Educational psychology, learning, teacher training. 


\section{La relación Teoría y Práctica en la Psicología de la Educación: consecuencias en la formación del educador}

\section{Resumen}

Este trabajo tiene el objetivo de discutir la relación teoría y práctica en el campo de la Psicología de la Educación y sus consecuencias para la formación del educador a partir del análisis de los trabajos presentados en las reuniones anuales de la ANPEd (especialmente en el Grupo de Trabajo Psicología de la Educación (GT20). El estudio contempló la lectura de trabajos encargados, comunicaciones y posters, presentados en las reuniones anuales de la ANPEd, en el período de 1998 a 2009. Los estudios presentados en la última década señalan que existe una aplicación de la psicología en la escuela, que básicamente es alimentada por la Psicología del Aprendizaje. Los estudios sobre subjetividad, identidad y constitución del sujeto indican una preocupación con el ser humano (constitución de la subjetividad), sin embargo aún con el enfoque muy psicológico. Al parecer todavía no superamos la dificultad de pensar la subjetividad en términos de contextos más amplios. Y lo que prevalece en estos estudios, son abordajes teóricos de la psicología del desarrollo. Otro bloque de estudios, apoyados principalmente en la incorporación de contribuciones de la psicología social indican la posibilidad de ver/pensarse la escuela. Sin embargo, aquí la restricción aún es de orden más conceptual. O sea la dicotomía permanece. Finalmente, identificamos una línea que debería o podría constituirse en la crítica epistemológica de toda esta construcción, pero todavía se encuentra muy presa a abordajes; se podría partir de una visión más contextualizada, superando el debate enfocado en abordajes. Consideramos que es necesario pensar la psicología más allá del territorio de autores o de áreas (como la psicología del aprendizaje, del desarrollo). Tal vez nuestro movimiento necesite redirigirse, trayendo las contribuciones de la psicología para una visión más amplia, inclusive de escuela (que permita comprender la escuela y sus actores en todas sus complejas dimensiones). Escuela no es sólo espacio de aprendizaje o de desarrollo, o de formación de profesores.

Palabras-clave: Psicología educacional, aprendizaje, formación de profesores.

\section{Introdução}

O objetivo deste trabalho é problematizar a relação entre teoria e prática no campo da Psicologia da Educação e suas implicações para a formação do educador. Para tanto, há que se pensar em algumas questões/teses: a Psicologia da Educação constitui-se em campo de conhecimento? Há uma teoria da Psicologia da Educação? E, ainda, se e como a Psicologia da Educação, enquanto um campo de conhecimento, com suas especificidades, princípios e objeto pode contribuir na formação de professores para a educação básica no Brasil?

Venho acompanhando a consolidação do Grupo Psicologia da Educação desde sua criação. Em 2005, realizamos um trabalho, no qual, Souza e eu analisamos a produção do GT 20 até aquele momento, com o intuito de mapear a produção científica apresentada no GT. O estudo envolveu a leitura de 16 trabalhos encomendados, 83 comunicações e 30 pôsteres, apresentados no período de constituição do Grupo de Estudo e de Trabalho Psicologia da Educação (1998-2004). Para as análises foram utilizadas fichas analíticas elaboradas nos moldes metodológicos de OZELLA (1998), com o intuito de mapear os trabalhos sob diferentes perspectivas (modalidade de trabalho, ano de apresentação, autor, instituição, programa de pós-graduação, titulação, temática, filiação teórica, objetivos, procedimentos metodológicos, resultados e observações). Foram utilizados, também, quadros-síntese com o objetivo de identificar a temática, filiação teórica, problema/problemática, resultados, metodologia, objetivo, interface com a educação e interface com a psicologia.
Na época, os resultados indicaram três tendências:

Na primeira tendência foram incluídos trabalhos nos quais a psicologia vai à educação para analisar aspectos do fenômeno educacional e volta às teorias psicológicas para compreendê-lo, fornecendo contribuições tanto à psicologia como à educação, ou, como afirma Gatti, enfoca os "... fenômenos educacionais vistos em sua base psicológica" (GATTI, 1997, p.81).

De acordo com nossa análise, na época, os trabalhos encomendados (que se pautavam na discussão das diferentes abordagens psicológicas) reiteravam esta tendência.

"Se por um lado, tais abordagens ainda refletem fortemente o lugar da Psicologia no campo educacional, por outro lado, é fundamental a demarcação de tais espaços e concepções para avançarmos na direção da construção do campo de conhecimento denominado 'Psicologia da Educação"' (SCHLINDWEIN, SOUZA et alii).

$\mathrm{Na}$ segunda tendência, foram incluídos trabalhos que realizavam uma "... análise crítica das apropriações de algumas teorias psicológicas pela Educação e que produziram um movimento de psicologização da educação, e a necessidade da constituição de abordagens críticas no campo da Psicologia da Educação, que superassem a primazia do conhecimento psicológico sobre outras modalidades de conhecimento" (SCHLINDWEIN, SOUZA et alii). Nestes trabalhos, o que prevalecia era uma espécie de movimento no 
qual a psicologia da educação critica o uso da psicologia na educação.

Neste grupo, inserem-se os trabalhos de Leandro Lajonquiére intitulado "As apropriações das teorias psicológicas pela prática educativa contemporânea: a Psicanálise frente à psicologização do cotidiano escolar"; Newton Duarte com a apresentação "As apropriações das teorias psicológicas pela prática educativa contemporânea: a incorporação de Piaget e de Vigotski ao ideário pedagógico"; Odair Sass com o artigo "Interrogações da Educação sobre a Psicologia da Educação"; Marília Gouveia de Miranda com o trabalho "Psicologia da Educação e Política Educacional" e Antônio Flávio Barbosa Moreira, com "Por entre ficções e descentramentos: discussões atuais de currículo e a Psicologia da Educação".

Uma terceira tendência compreendia estudos sobre a subjetividade (especialmente do professor) que, na época foram considerados indicativos de uma confirmação do campo da psicologia da educação.

Por exemplo, Rey (2001) concebe a educação enquanto um dos espaços privilegiados de constituição da subjetividade e do desenvolvimento humano. Aponta a questão da subjetividade como um fenômeno individual e social que, portanto, exige uma abordagem metodológica que dê conta da complexidade dos processos de sua constituição. Nessa perspectiva, a constituição da subjetividade no processo educativo deve ser analisada por meio da compreensão dos processos de atribuição de significado e de sentido constituídos nos espaço social mais amplo.

Em 2009, foi realizado um novo trabalho, com o intuito de mapear outros cinco anos de produção do GT. Foram analisadas 58 comunicações apresentadas no grupo, no período compreendido entre os anos de 2005 e 2009. Reiteramos a idéia apresentada em 2005, de que as comunicações “... expressam a produção recente acadêmicocientífica na interface Psicologia e Educação dos programas de Educação e Psicologia Escolar/Educacional no Brasil. As comunicações são indicativas do estado do conhecimento no campo da Psicologia da Educação" (SOUZA et alii, 2005). Entretanto, diferentemente do trabalho apresentado em 2005, debruçamo-nos sobre as temáticas, resumos e referenciais teóricos utilizados nas comunicações.

Em nossas análises, Cordeiro e eu consideramos que não houve grandes alterações, em termos de temáticas apresentadas, em relação ao primeiro estudo. Entretanto, como utilizamos uma metodologia diversa daquela do primeiro trabalho, nossas análises permitiram a construção de 4 categorias: Cognição e Afetividade nos Processos de Aprendizagem (abordada de maneira geral, conteúdos específicos e aprendizagem do professor); Desenvolvimento, subjetividade e identidade; Processos psicossociais e, Fundamentos teóricos e epistemológicos da Psicologia da Educação - interface da Psicologia e Educação.

A categoria 'Cognição e afetividade nos processos de aprendizagem' reuniu os trabalhos que utilizaram a psicologia como ferramenta para a compreensão dos fenômenos educacionais, mantendo uma das tendências observada no estudo anterior. Procuramos, entretanto, detalhar melhor quais os problemas abordados nesta temática. Constatamos que a grande parte de trabalhos desta temática $(n=15 / 58$ $25 \%$ ) discutem a cognição e/ou afetividade nos processos de aprendizagem. Encontramos, também, quatro trabalhos que se referem à aprendizagem do professor e apenas 2 trabalhos que discutem esta temática com referência a conteúdos específicos.

Consideramos que este resultado pode ser analisado sob diferentes perspectivas. Uma delas diz respeito à existência, na reunião da ANPED, de grupos de trabalho, cujo foco são conteúdos específicos, como, por exemplo, os GT's Educação Matemática, Alfabetização, Leitura e Escrita, Educação e Arte, ou ainda, o de Formação de Professores.

Foi possível perceber que os focos apresentados nas comunicações, dentro desta temática, são predominantemente situados no contexto escolar. Este fato reitera a preocupação em utilizar os conhecimentos da psicologia para compreender fenômenos educacionais, mais especificamente aqueles relacionados com a aprendizagem.

Em nossas análises, outra categoria que também prevalece no estudo realizado em 2005 é 'Desenvolvimento, subjetividade e identidade'. Esta categoria foi constituída por 16 trabalhos, dos quais 7 são reflexões teóricas; 8 são comunicações que enfocam o contexto escolar, e 1 trabalho trata da inclusão. Estes trabalhos buscam subsídios, sobretudo, na psicologia do desenvolvimento, em diferentes abordagens (predominantemente histórico-cultural e psicanalítica). São trabalhos que olham para a escola enquanto um contexto de desenvolvimento dos sujeitos (crianças, adolescentes e adultos professores).

Ainda neste estudo apresentado em 2009, incluímos uma categoria que não tinha sido evidenciada no estudo anterior: "Processos psicossociais'. Engloba comunicações que discutem os contextos educacionais com uma predominância das contribuições da psicologia social. Estas contribuições trazem uma nova possibilidade de interface entre a psicologia e a educação, e permitem ver a escola dentro de uma realidade social mais ampla, sem perder de foco os sujeitos (atores nestes contextos). Os fenômenos educacionais são descritos considerando os sujeitos investigados nas intrincadas relações que se estabelecem nos contextos, sejam eles relacionais, escolares ou familiares (e as respectivas representações).

Na categoria 'Fundamentos teóricos e epistemológicos da Psicologia da Educação' - interface entre Psicologia e Educação - percebemos que as comunicações são constituídas por estudos fundamentalmente teóricos, no sentido de que não se tratam de pesquisas empíricas. Nove dentre estes estudos discutem a interface da psicologia com a educação, sobretudo nas perspectivas histórico-cultural e psicanalítica. Os outros quatro estudos desta categoria constituem-se em exercícios de contextualização das teorias psicológicas em problemáticas escolares (tecnologias, políticas, autoridade e concepção de criança escolarizada), com o intuito não apenas de confirmar a teoria, mas também de apresentar elementos para ampliar o campo da psicologia da educação. 
Portanto, são estudos que contribuem para a afirmação do campo da psicologia da educação, fazendo uma análise critica da aplicação das teorias (ou dos discursos) psicológicas ao campo da educação (13/58).

Faço esta rápida contextualização para destacar que, para o presente trabalho, eu retornei à leitura das 144 comunicações apresentadas no GT Psicologia de Educação, desde sua criação, no ano de 1998, com o objetivo de analisar as problemáticas investigadas (os objetos de investigação). Então, minha discussão aqui tem como ponto de partida e referência, a produção qualificada discutida no GT Psicologia da Educação nestes últimos doze anos. E é desde este lugar que proponho uma problematização a respeito da Psicologia da Educação

Em primeiro lugar, cabe questionar: a Psicologia da Educação constitui-se em um campo de conhecimento? Muitos estudos têm sido apresentados, especialmente nas últimas décadas, no Brasil, discutindo a relação que se estabelece entre Psicologia e Educação e como a interrelação destas duas áreas de conhecimento pode se constituir em um campo híbrido com objeto próprio (ainda que apoiado, muitas vezes tendenciosamente, em uma ou outra área).

Por um lado, trabalhos de autores como Maria Regina Maluf (1996, 2004), Miriam Warde (1996), Bernardete Gatti (1997), Marília Gouveia de Miranda (2006, 2008) têm problematizado esta discussão, evidenciando que não se trata de uma questão resolvida.

Ao mesmo tempo, a área convive com manuais que divulgam o que seria uma proposta de abordagem deste campo. Por exemplo, os trabalhos publicados por Davis \& Oliveira e os de César Coll (apenas para citar alguns).

Miranda afirma que "... a psicologia vem reiteradamente se firmando como fundamento do aparato teóricometodológico que constitui a prática educativa formal e informal" (MIRANDA, 2006). Concordamos com a autora que a relação que se estabelece entre psicologia e educação, no tratamento das mais diferentes questões educacionais está muito mais articulada do que se pode pensar em um primeiro momento. Diríamos que é uma relação quase visceral. É impossível pensar em alfabetização, infância, ensino, aprendizagem, por exemplo, sem ter alguma relação ou apoio em algum aporte da psicologia, ainda que este não seja explicitado.

Ao retomar a análise dos focos de investigação dos 144 trabalhos apresentados no GT 20, acabei constituindo 4 blocos categoriais para a presente apresentação, ou seja, a relação teoria-prática na psicologia da educação.

O primeiro bloco está constituído por trabalhos que partem de algum referencial da psicologia e têm na educação a possibilidade de discussão empírica deste referencial de base (teórico-metodológico). Foram discutidos 95/144 $(66 \%)$ trabalhos com este formato metodológico, em diferentes abordagens psicológicas, com predominância da psicologia histórico-cultural, da psicanálise e da abordagem comportamental.

Exemplos:
ELEMENTOS PARA UMA PROPOSTA DE FORMAÇÃO DE PROFESSORES A PARTIR DA ANÁLISE COMPORTAMENTAL DE B.F.SKINNER; NÍVEIS DE AQUISIÇÃO DA PROFISSIONALIDADE DOCENTE: CONTRIBUIÇÕES DE UMA LEITURA PIAGETIANA (CHAKUR, 2004); AVALIAÇÃO DINÂMICA: UMA PROPOSTA ALTERNATIVA E COMPLEMENTAR DE AVALIAÇÃO COGNITIVA EM CRIANÇAS COM INDICAÇÃO DE DIFICULDADE DE APRENDIZAGEM (DIAS \& ENUMO, 2007).

Em um segundo bloco, incluí os trabalhos que partem de uma problemática educacional, apóiam-se em referencias da psicologia para as discussões e análises e tentam, de alguma forma, apresentar indicadores ou propostas para a educação. Ou seja, são trabalhos que partem da educação, bebem da psicologia (especialmente da psicologia do desenvolvimento e da aprendizagem) e retornam para a educação. Foram apresentados 18/144, ou seja, 12,5\% dos trabalhos nesta modalidade.

Exemplos:

CONCEPÇÕES DE PROFESSORES SOBRE QUESTÕES RELACIONADAS À VIOLÊNCIA NA ESCOLA(LOBATO,2006); POSSIBILIDADES DE VIVER A INFÂNCIA: UM ESTUDO A PARTIR DA ÓTICA DE CRIANÇAS ENTRE 5 E 12 ANOS(FRANCO, 2009); UMA QUESTÃO PARA A EDUCAÇÃO INCLUSIVA: "EXPOR-SE OU RESGUARDAR-SE?"( SEKKEL, 2009).

26/144 trabalhos (18\%) foram problematizados a partir de uma relação intrincada entre a psicologia e a educação, constituindo suas problemáticas de investigação em um campo híbrido que articula psicologia e educação: ou seja, um campo de conhecimento que pode ser denominado Psicologia da Educação. Consideramos que, neste campo, a problemática de estudo somente poderia se constituir no espaço educacional ou escolar e em uma abordagem psicológica que considere os aspectos sociais, culturais, afetivos, na constituição da pessoa ou dos grupos.

Consideramos que estes estudos são indicativos de uma Psicologia da Educação. A problemática e o método se articulam de forma que aportes teóricos e metodológicos da educação e da psicologia se constituam de forma indissociável.

Exemplos:

RACISMO, PRECONCEITO E DISCRIMINAÇÃO ÉTNICOS NA EDUCAÇÃO INFANTIL; CONSTITUIÇÃO DA SUBJETIVIDADE NAINFÂNCIA; AINTERAÇÃO NAESCOLA E SEUS SIGNIFICADOS E SENTIDOS NA FORMAÇÃO DE VALORES: UM ESTUDO SOBRE O COTIDIANO ESCOLAR (SOUZA, 2004); A PSICOLOGIA E A FORMAÇÃO DE PROFESSORES: AÇÃO E REFLEXÃO A PARTIR DA PERCEPÇÃO DE PROFESSORES EM FORMAÇÃO. 
Entretanto não é fácil definir ou determinar uma teoria da psicologia da educação. O que definiria e delimitaria uma teoria da Psicologia da Educação?

Esta é uma pergunta que não se pode responder de forma direta.

Mas, podemos afirmar que a Psicologia sempre se constituiu em fundamento para o campo da Educação. E que, como área reconhecida de conhecimento, tem, na Educação um campo fértil para o desenvolvimento de estudos.

Entretanto, o que pretendo problematizar é a lacuna que se estabelece na produção de um conhecimento que declaradamente suporte estudos do campo da Psicologia da Educação, como área do conhecimento.

Poderíamos pensar que Piaget, por exemplo, propiciou grande contribuição para os estudos da Psicologia de Desenvolvimento Humano; bem como Vigotski e seus colaboradores, propondo uma psicologia histórico-cultural, que permite compreender a constituição do homem. Ambos, Piaget e Vigotski possuem trabalhos acerca da relação profícua que se estabelece entre a pedagogia e a psicologia. $\mathrm{E}$ muitos outros poderiam ser citados. Mas, ainda assim, não chegamos a uma teoria que aborde de forma mais totalizante a questão da psicologia da educação em toda a sua complexidade. E, menos ainda, em uma superação da relação teoria e prática para a problemática educacional e a formação de professores...

Então, chegamos a nossa última questão: como a Psicologia da Educação, enquanto campo de conhecimento, com suas especificidades, princípios e objeto pode contribuir na formação de professores para a educação no Brasil?

Consideramos que a psicologia constitui-se em uma das disciplinas que deveria fundamentar a formação dos professores, especialmente no que se refere aos processos de constituição do ser humano. Concordamos com Raposo (em trabalho apresentado em 2006, no GT), quando afirma que as disciplinas de psicologia “... representam um eixo importante na formação do professor e, como tal, devem partir das questões educacionais, tornando-as objeto de investigação, e analisá-los (estes objetos) nas perspectivas dos conteúdos e métodos psicológicos, com foco no retorno ao ponto de partida que é, afinal, a prática educativa." Para a autora, os campos da psicologia e da educação constituemse em unidades dialéticas de ação e reflexão, favorecendo as tomadas de decisões dos professores, na perspectiva do bom desempenho de seus alunos.

A idéia é que não só os conceitos sejam ensinados nos cursos de formação de professores, mas que estes conceitos sejam dinamizados em uma perspectiva metodológica que favoreça a compreensão e análise dos fenômenos educacionais.

Em outras palavras, uma formação consistente no campo da psicologia da educação poderia instrumentalizar os professores para uma postura profissional mais crítica e engajada. Não se trata de aprender, no campo da psicologia, alguns conceitos, mas de valer-se dos referenciais metodológicos que propiciem a compreensão do fenômeno educacional, de forma articulada.
Consideramos que a disciplina psicologia, como elemento fundante nos cursos de pedagogia e de formação de professores, possa constituir-se em uma ferramenta efetiva aliada a prática pedagógica. Concordamos com Pereira, Almeida e Azzi (2002) quando afirmam que a teoria toma outra forma na medida em que favorece a leitura da experiência vivida, possibilitando o esclarecimento da situação vivenciada.

Em pesquisa recente, Gatti e Barreto (2009) apresentam uma experiência interessante, na UFF em parceria com a Prefeitura de Angra dos Reis, cujo diferencial, em termos de constituição do curso, é desenvolver uma postura quanto à aquisição de conhecimento dos futuros professores, concebida como uma busca permanente, admitindo-o como algo prático, que se constrói pela atividade dos sujeitos, os quais, pela sua experiência, se relacionam com os objetos (GATTI \& BARRETO, 2009, p. 120). Nesta experiência, pretende-se que a prática social direcione a prática pedagógica, em um movimento que pretende superar a fragmentação que as disciplinas de filosofia, psicologia e sociologia, de maneira geral, cumprem nos cursos de formação de professores.

Ou seja, podemos citar algumas saídas ou experiências interessantes e inovadoras, que promovem ou tentam promover certa superação da fragmentação teoria e prática. Entretanto, de modo geral, o que temos acompanhado é uma espécie de encolhimento das disciplinas de psicologia nos cursos de formação de professores e, ainda, uma abordagem desta disciplina, no contexto dos cursos, muito teórica e desvencilhada da prática.

O GT 20, apesar de sua produção qualificada, ainda apresenta uma contribuição tímida para a formação de professores na perspectiva de uma superação da dicotomia teoria e prática.

Em síntese, poderíamos pensar em algumas perspectivas ou saídas para o campo de estudos da Psicologia e da Educação:

Os estudos apresentados nesta última década são indicativos de que temos uma aplicação da psicologia na escola que basicamente é alimentada pela psicologia da aprendizagem.

Os estudos sobre subjetividade, identidade e constituição do sujeito indicam uma preocupação com o ser humano (constituição da subjetividade), mas ainda com o enfoque muito psicológico. Ao que parece, ainda não superamos a dificuldade de se pensar esta subjetividade em termos de contextos mais amplos. E, o que prevalece, nestes estudos, são abordagens teóricas da psicologia do desenvolvimento.

Outro bloco de estudos, apoiados principalmente na incorporação de contribuições da psicologia social são indicadores de uma possibilidade de olhar/se pensar a escola. Entretanto, aqui, a limitação ainda é de ordem mais conceitual. Ou seja, a dicotomia permanece.

Finalmente, identificamos uma linha que deveria ou poderia se constituir na crítica epistemológica de toda esta construção; mas que ainda está muito presa a abordagens; poder-se-ia partir de uma visão mais contextualizada, superando o debate focado em abordagens. Consideramos que 
é preciso pensar a psicologia para além do território de autores ou de áreas (como a psicologia da aprendizagem, do desenvolvimento). Talvez nosso movimento precise ser redirecionado, trazendo as contribuições da psicologia para uma visão mais ampla, inclusive de escola (que permita compreender a escola e seus atores em todas as suas complexas dimensões). Escola não é só espaço de aprendizagem, ou de desenvolvimento, ou de formação de professores... É algo mais complexo, que precisa ser tomado como tal.

\section{Referências}

Chakur, Cilene Ribeiro de Sá Leite. NÍVEIS DE AQUISIÇÃO DA PROFISSIONALIDADE DOCENTE: CONTRIBUIÇÕES DE UMA LEITURA PIAGETIANA. http://www.anped.org.br/reunioes/23/ textos/2004t.PDF

DAVIS,Claudia e OLIVEIRA,Zilma de. Psicologia na educação. Editora Cortez.São Paulo.1990.

DIAS, Tatiane Lebre e ENUMO, Sônia Regina Fiorim. AVALIAÇÃO DINÂMICA: UMAPROPOSTAALTERNATIVAE COMPLEMENTAR DE AVALIAÇÃO COGNITIVA EM CRIANÇAS COM INDICAÇÃO DE DIFICULDADE DE APRENDIZAGEM, 2007. http://www. anped.org.br/reunioes/27/gt20/t2012.pdf

DUARTE, N. As apropriações das teorias psicológicas pela prática educativa contemporânea: a incorporação de Piaget e de Vigotski ao ideário pedagógico. In: 23 reunião da ANPED, 2000, Caxambu. Anais da 23 reunião anual da ANPED, 2000.

FRANCO, Márcia Elisabete Wilke. POSSIBILIDADES DE VIVER A INFÂNCIA: UM ESTUDO A PARTIR DA ÓTICA DE CRIANÇAS ENTRE 5 E 12 ANOS, 2009. http://www.anped.org.br/ reunioes/32ra/arquivos/trabalhos/GT20-5397--Int.pdf

GATTI, B. A. O que é Psicologia da Educação? Ou o que ela pode vir a ser como área de conhecimento?. Psicologia da Educação, São Paulo, SP, n. 5, p. 73-90, 1997.

GATTI, Bernadete Angelina e BARRETO, Elba Siqueira de Sá (Coord.). Professores do Brasil: impasses e desafios. Brasília: UNESCO, 2009.

LAJONQUIÈRE, L. As apropriações das teorias psicológicas pela prática educativa contemporânea: a psicanálise frente à psicologização do cotidiano escolar. In: 23 reunião da ANPED, 2000. Anais da 23 reunião anual da ANPED, 2000)

LOBATO, Vivian da Silva. CONCEPÇÕES DE PROFESSORES SOBRE QUESTÕES RELACIONADAS À VIOLÊNCIA NA ESCOLA,2006. http://www.anped.org.br/reunioes/29ra/trabalhos/ trabalho/GT20-1739--Int.pdf

MALUF, M. R. (Org.). Psicologia Educacional. Questões contemporâneas. 1a. ed. São Paulo: Casa do Psicologo, 2004.
MALUF, M. R. A Formação Profissional do Psicólogo Brasileiro Psicologia. Teoria e Pesquisa, São Paulo, v. 01, n. 01, p. 31-45, 1996.

MIRANDA, M. G. \& RESENDE, A. C. A. (orgs.). Escritos de Psicologia, Educação e Cultura. Goiânia: Ed. Da UCG, 2008.

MIRANDA, Marília G. de. O Professor Pesquisador e Sua Pretensão de Resolver a Relação Entre a Teoria e a Prática na Formação de Professores. In: O Papel da pesquisa na formação e na prática dos professores. Campinas: Papirus, 5 ed, 2006.

MOREIRA, A. F. B. Por entre ficções e descentramentos: discussões atuais de currículo e a Psicologia da Educação. In: 26 reunião da ANPED, 2003, Caxambu. Anais da 26 reunião anual da ANPED, 2003).

OZELLA, Sergio . Vinte anos de Psicologia/Psicologia Social na América Latina. Psicologia Revista, São Paulo, v. 6, p. 31-67, 1998.

Pereira, M.A. L.,Almeida, P. C.A. eAzzi, R. G. (2002)Adimensãoteóricoprática da psicologia educacional na formação de professores: a metodologia da problematização como desencadeadora da articulação entre teoria e pratica. In: Azzi, R. G. e Sadalla, A. M. F. A. (org.) Psicologia e formação docente: desafios e conversas (pp. 185-211). São Paulo: Casa do Psicólogo.

Raposo, M.B.T. e Maciel, D. (2005) As interações professor-professor na co-construção dos projetos pedagógicos na escola. Em: Psicologia: Teoria e Pesquisa. 21, (3), 309-317

Rey, Fernando. A pesquisa e o tema da subjetividade em educação. XXIV Reunião Anual da ANPED. 2001. http://www.anped.org.br/ reunioes/24/te.htm

SASS, O. Interrogações da Educação sobre a Psicologia na Educação brasileira. In: 24 reunião da ANPED, 2001, Caxambu. Anais da 24 reunião anual da ANPED, 2001.

SEKKEL, Marie Claire. UMA QUESTÃO PARA A EDUCAÇÃO INCLUSIVA: “EXPOR-SE OU RESGUARDAR-SE?”, 2009. http:// www.anped.org.br/reunioes/32ra/arquivos/trabalhos/GT20-5438Int.pdf

SOUZA, Vera Lucia Trevisan de. A INTERAÇÃO NA ESCOLA E SEUS SIGNIFICADOS E SENTIDOS NA FORMAÇÃO DE VALORES: UM ESTUDO SOBRE O COTIDIANO ESCOLAR, 2004. http://189.1.169.50/reunioes/27/gt20/t2013.pdf

WARDE, Mirian Jorge. Psicologia e Educação: a produção discente na pós-graduação em educação no Brasil (1982-1991). REVISTA DE PSICOLOGIA DA EDUCAÇÃO, SÃO PAULO, v. 1, n. 1, p. 4361, 1996. 
Recebido em: 18/10/2010

Aprovado em: 08/12/2010

Sobre autora

Luciane Maria Schlindwein (lucmas@uol.com.br)

Universidade Federal de Santa Catarina, Florianópolis - SC.

\section{Correspondência}

Rua Servidão Alcides Anacleto Vieira,93, apto. 401.

Bairro Pantanal - Florianópolis - SC

CEP 88040-360 